\title{
Poisson logit hurdle model with associated factors of perinatal mortality in Ethiopia
}

\author{
Abiba Mihret Aragaw $^{1 *} \mathbb{D}$, Abebaw Gedef Azene ${ }^{2}$ and Mekuanint Simeneh Workie ${ }^{3}$
}

\section{*Correspondence:}

abibami24@gmail.com

1 Department of Statistics,

College of Natural

and Computational Science,

Debre Markos University,

Debre Markos, Ethiopia

Full list of author information

is available at the end of the

article

\begin{abstract}
Perinatal mortality is the total number of fetal death and early neonatal death. Perinatal mortality is a major public health problem, particularly in developing countries, and is used as an implication of the economic, social, and health status of the country. The analysis of count data with hurdle and zero-inflated count models are the most applicable methods to accommodate with excessive zero counts. Therefore, this study aimed to apply the Poisson logit hurdle model to identify the associated factors of perinatal mortality in Ethiopia. A cross-sectional study design was conducted in Ethiopia using EDHS 2016. The sample was multistage stratified and units selected in a two-stage cluster sampling design. The association between the outcome and the independent variables was determined using the Poisson logit hurdle model. A total of 7230 mothers were obtained from EDHS 2016 survey. Of these mothers, 95.27\% of them never, $4.47 \%$ of them once, $0.26 \%$ twice, and $0.04 \%$ three times experienced perinatal mortality preceding 5 years of the survey. The main protective associated factors were 40-49 years age of mother, having long preceding birth interval, and secondary + husband education. Parity is greater than four, rural residence, Caesarean section delivery, multiple pregnancies, institutional delivery, having a history of abortion were increased perinatal mortality per mother. This study implies that intervention is needed on family planning and mode of delivery to minimize perinatal mortality in the country.

Keywords: Perinatal mortality, Associated factors, Poisson logistics hurdle model, Ethiopia
\end{abstract}

\section{Introduction}

Perinatal mortality is that the total number of deaths within the perinatal period. This includes stillbirth (fetal death) and early neonatal death (ENND) i.e., death of live newborns before the age of 7 completed days. Perinatal mortality rate (PMR) is calculated as a proportion of the overall number of perinatal deaths per total number of births [1-3]. This perinatal period is the most critical in an individual's life, and also the rate of death during this period is higher than any other period of life. Perinatal mortality is especially associated with maternal conditions during late pregnancy and intrapartum conditions. Perinatal mortality reflects the quality of delivery care and it is also a key indicator of socioeconomic development and also the overall health status of any country [4].

(c) The Author(s) 2022. Open Access This article is licensed under a Creative Commons Attribution 4.0 International License, which permits use, sharing, adaptation, distribution and reproduction in any medium or format, as long as you give appropriate credit to the original author(s) and the source, provide a link to the Creative Commons licence, and indicate if changes were made. The images or other third party material in this article are included in the article's Creative Commons licence, unless indicated otherwise in a credit line to the material. If material is not included in the article's Creative Commons licence and your intended use is not permitted by statutory regulation or exceeds the permitted use, you will need to obtain permission directly from the copyright holder. To view a copy of this licence, visit http:// creativecommons.org/licenses/by/4.0/. 
Globally, over 5 million perinatal deaths occur every year, ending preventable perinatal deaths will continue to be a significant part of the global public health agenda beyond 2015. In developed countries, perinatal mortality is a rare event. Ninety-seven percent of globally reported stillbirths and $98 \%$ of neonatal deaths occurred in developing countries [5]. Perinatal mortality is a major public health problem, particularly in developing countries, and has huge economic, social, and health implications for families and nations. As a result, reducing stillbirths and early neonatal deaths continued to be an important part of the third Sustainable Development Goal (SDG-3), to end preventable child deaths by $2030[2,6]$. The highest mortality rate is found in sub-Saharan Africa. PMR and intra partum stillbirths are 5 and 14 times higher in developing regions compared with developed regions consecutively [7].

The analysis of count data with many zeros can be done in various fields, including medical and public health. Several models have been developed in recent decades to analyze these count data like perinatal mortality data [8]. The usual Poisson regression model and the Negative Binomial model were not significant in explaining and handling over-dispersion due to excess zeros [9]. Therefore, Hurdle and zero-inflated count models are the most applicable methods to deal with count data having excessive zero counts [10]. Zero-inflated models and hurdle models provide a way of modeling the excessive proportion of zero values and allow for over-dispersion [11-13]. The choice between the zero-inflated model and the hurdle model is often dependent on the nature of the data. Although these two models are similar in many aspects, conceptually there is a little difference between the two models depending on the application and the data collection. The main difference zero inflated and hurdle models are generally used in the setting of excess zeroes. Zero inflated models are typically used if the data contains excess structural and sampling zeros, whereas hurdle models are generally used when there are only excess sampling zeroes.

The hurdle model includes a mass at zero and a truncated distribution whereas the zero-inflated model is based on a mass at zero and a regular distribution even if the inferential results are often very similar. Hurdle models are more general in the sense that they can handle both zero deflation and zero inflation data $[14,15]$. The hurdle models do not necessarily have to be set at zero. Zero-inflated models are less general than hurdle models due to the assumption that two different types of zeros (structural or true zeros, vs. sampling zeros) may exist in the data and it handles only zero inflation data [14]. Ideally, the hurdle models are more appropriate for cases where a real separation of mechanisms producing the zeros and the positive counts is justified [16].

In many cases because of many zeros in the dependent variable, the mean is not equal to the variance value of the dependent variable. Due to that, the Poisson model is no longer suitable for this kind of data. The hurdle model is better than the zero inflated models in cases of zero excess data there will zero deflation could still occur at specific levels of covariates $[14,15,17]$. To reduce mortality, determinants of perinatal mortality at country context initiatives, which focus on encouraging evidence-based advocacy and effective interventions targeting perinatal mortality reduction, through local decision making.

In Ethiopia, there are a few studies that have investigated the associated factors of perinatal mortality using a simple logistic regression model. But as far as our search, this 
is the first study in Ethiopia, which applied appropriate statistical methods to measure the associated factors using the Poisson logit hurdle model using the latest round demographic health survey data (EDHS 2016). Therefore, this study aimed to apply the Poisson logit hurdle model to identify the main associated factors of perinatal mortality in Ethiopia.

\section{Method}

\section{Study design and source of data}

The dataset used for this study was obtained from 2016 Ethiopian Demographic Health Surveys conducted from January 18 to June 27, 2016, across the country. The survey was a population-based cross-sectional study. For the surveys, the 2016 EDHS sample was stratified and selected in two stages. In the first stage, a total of 645 clusters were randomly selected proportional to the household size from the sampling strata, and in the second stage, 28 households per cluster were selected using systematic random sampling. In this survey, a total of 7230 mothers selected from 645 clusters were included in this study.

\section{Study variable}

\section{Dependent variable}

Number of perinatal mortality per mother preceding 5 years from the survey.

\section{Independent variable}

Place of residence, Age of mothers (years), Educational status of mothers, Educational status of husbands, mode of delivery, place of delivery, ANC visit, Parity, Type of birth, and history of abortion.

\section{Operational definition}

Perinatal mortality is deaths after 28 competed for gestational weeks (still birth) and the first 7 days of birth (early neonatal death) per mother preceding 5 years from the survey.

Antenatal care visit is the number of attending ANC clinic during pregnancy, which categorizes the mother who visited an ANC clinic at least four times $(\geq 4)$, the mother who visited an ANC clinic at most three times $(<4)$.

Parity is the number of life born babies of the mother. The categories are: the mother who had given at least four life birth $(\geq 4)$ and the mother who had given at most three life birth $(<4)$.

Mode of delivery is the mode of giving the last birth preceding 5 years from the survey. Categories are: caesarean section (CS) and normal (vaginal).

History of abortion is the history of terminated pregnancy of the mother. Categories: Yes/No.

\section{Statistical method}

In this study, the variable of interest is a count variable. When the dependent variable is a count, it is appropriate to use non-linear models based on non-normal distribution to describe the relationship between the response variable and a set of predictor variables. For count data, the standard framework for explaining the relationship between 
the outcome variable and a set of explanatory variables includes the Poisson, ZIP, and Poisson hurdle models. The advanced models for this study count data are the Poisson hurdle model.

\section{Poisson regression model}

Poisson regression has been widely used for fitting count data. It is traditionally conceived as the basic count model upon which a variety of other count models are based [10]. The probability mass function for Poisson regression is:

$$
\mathrm{P}(\mathrm{Yi}=\mathrm{yi})=\frac{\mathrm{e}^{-\mu_{\mathrm{i}}} \mu_{i}^{y_{i}}}{\mathrm{y}_{\mathrm{i} !}}, \quad y_{i}=0,1,2,3,4, \ldots
$$

$\mu_{\mathrm{i}}$ is the parameter of the Poisson distribution. It can be proved that the Poisson distribution is the mean equal to the variance. Such that the Poisson regression model is:

$$
\log \left(\mu_{\mathrm{i}}\right)=\beta_{0}+\beta_{1} x_{1}+\beta_{2} x_{2}+\beta_{3} x_{3}+\cdots+\beta_{p} x_{p}=X_{i}^{T} \beta
$$

$\beta$ are the vector coefficients $X_{i}^{T}$. Unfortunately, in much of the cases, the number of perinatal mortality data produces variance that is greater than the mean, well known as over-dispersion. The over-dispersion is a result of extra variation in the number of perinatal death means which can be caused by various factors such as model misspecification, omission of important covariates, and excess zero counts [18]. In this case, applying a Poisson regression model for the number of perinatal death data would result in an underestimation of the standard error of the regression parameters. Therefore, the negative binomial model will be introduced.

In some cases, excess zeros in the number of perinatal death data exist and are considered as a result of overdispersion. In this case, the NB model cannot be used to handle the overdispersion which is due to the high amount of zeros. To do this, zero-inflation (ZI) models including Zero Inflated Poisson (ZIP) models can be used. ZIP models assume that all zeros count come from two different processes: the process generating excess zero counts derived from a binary model, and the process generating non-negative counts for the number of perinatal death including zero values. A Poisson regression model with many zero outcomes on the response variable. The zero-inflated Poisson regression model is more effective for many zero outcomes than Poisson regression.

\section{Zero inflated Poisson regression model}

In ZIP regression, the counts $Y_{i}$ equal 0 with probability $\mathrm{p}_{\mathrm{i}}$ and follow a Poisson distribution with mean $\mu_{i}$, with probability $1-\mathrm{p}_{\mathrm{i}}$ where $\mathrm{i}=0,1,2, \ldots, \mathrm{n}$. ZIP model can thus be seen as a mixture of two-component distributions, a zero part, and no-zero components, given by [10]:

$$
\mathrm{P}(\mathrm{Y}=\mathrm{y})=\left\{\begin{array}{l}
p_{i}+\left(1-p_{i}\right) e^{-\mu_{i}}, \quad y_{i=0} \\
\left(1-p_{i}\right) \frac{e^{-\mu_{i}} \mu_{i}^{y_{i}}}{y_{i !}}, \quad y_{i}=1,2,3, \ldots
\end{array}\right.
$$

Assume that there are $\mathbf{p}$ predictors for logistic regression function and negative binomial regression function. Hence, the ZIP regression model can be written as follow: 
$\operatorname{Logit}\left(p_{i}\right)=x_{i}^{T} \beta$ and $\log \left(\mu_{i}\right)=x_{i}^{T} \gamma$ where $x_{i}-\left[1, x_{1}, x_{2} \ldots x_{p}\right]$ is $(\mathrm{p}+1) \times 1$ dimensional vector, $\beta=\left[\beta_{0}, \beta_{1}, \beta_{2}, \beta_{3} \ldots \beta_{p}\right]$ and $\gamma=\left[\gamma_{0}, \gamma_{1}, \gamma_{2}, \gamma_{3}, \ldots \gamma_{p}\right]$ is a $(\mathrm{p}+1) \times 1$ dimensional vector of regression parameters.

\section{Poisson hurdle regression model}

A hurdle model consists of two components-a point mass at zero and a distribution that generates non-zero counts. The first component is a binary component that generates zeros and ones (here "ones" correspond to non-zero values in data) and the second component generates non-zero values from a zero-truncated distribution. The most widely used hurdle models are those with the hurdle value at zero [4]. All zeros in the hurdle model are assumed to be "structural" zeros, i.e., they are generated from a single process, and are observed since the condition is absent. We explore two zero-truncated count distributions for the hurdle model specification [19]. The hurdle model of count data can be expressed as follows for the Poisson distribution. We consider a Hurdle Poisson Regression Model in which the response variable y has the distribution:

$$
\mathrm{P}(\mathrm{Y}=\mathrm{y})=\left\{\begin{array}{l}
p_{i}, y_{i=0} \\
\left(1-p_{i}\right) \frac{e^{-\mu_{i}} \mu_{i} y_{i}}{y_{i !}\left(1-e^{-\mu_{i}}\right)}, \quad y_{i}=1,2,3, \ldots
\end{array}\right.
$$

where $\mu_{i}$ is the mean of the untruncated Poisson distribution.

Zero and truncated hurdle model:

$$
\operatorname{Logit}\left(p_{i}\right)=x_{i}^{T} \beta \text { and } \log \left(\mu_{i}\right)=x_{i}^{T} \gamma
$$

where $x_{i}=\left[1, x_{1}, x_{2} \ldots x_{p}\right]$ is $(\mathrm{p}+1) \times 1$ dimensional vector, $\beta=\left[\beta_{0}, \beta_{1}, \beta_{2}, \beta_{3} \ldots \beta_{p}\right]$ and $\gamma=\left[\gamma_{0}, \gamma_{1}, \gamma_{2}, \gamma_{3}, \ldots \gamma_{p}\right]$ is a $(\mathrm{p}+1) \times 1$ dimensional vector of regression parameters.

The Maximum Likelihood Estimation (MLE) method is used to estimate parameters in the count models. This study was a Poisson logit hurdle to accommodate the excess zeros for the number of perinatal death count data. In this paper, Akaike's information criteria (AIC) and log-likelihood values are used for model selection measures. It is also used dispersion parameters to test for overdispersion. The generalized Pearson $\chi^{2}$ statistic which is the standard measure of goodness of fit is used to evaluate the sufficiency of the analyzing methods. Akaike's information criteria (AIC) and log-likelihood are basic methods of assessing the performance of the models and model selection [10].

\section{Result}

A total of 7230 mothers were obtained from EDHS 2016 survey. The frequency and percentage distribution of the number of perinatal mortality per mother in Ethiopia are presented in Fig. 1 based on information from 12,645 respondents. Of 7230 mothers, $95.27 \%$ of them never, $4.47 \%$ of them once, $0.26 \%$ twice, and $0.04 \%$ three times experienced perinatal mortality preceding 5 years of the survey. This indicates zero outcomes were large in numbers. However large numbers of perinatal mortality per mother are being observed less frequently. This leads to a positively skewed distribution. And also this excess zero leads that the data better to fitted by a zero-inflated and hurdle model which takes into account excess zeroes. A histogram is highly peaked at zero, we 


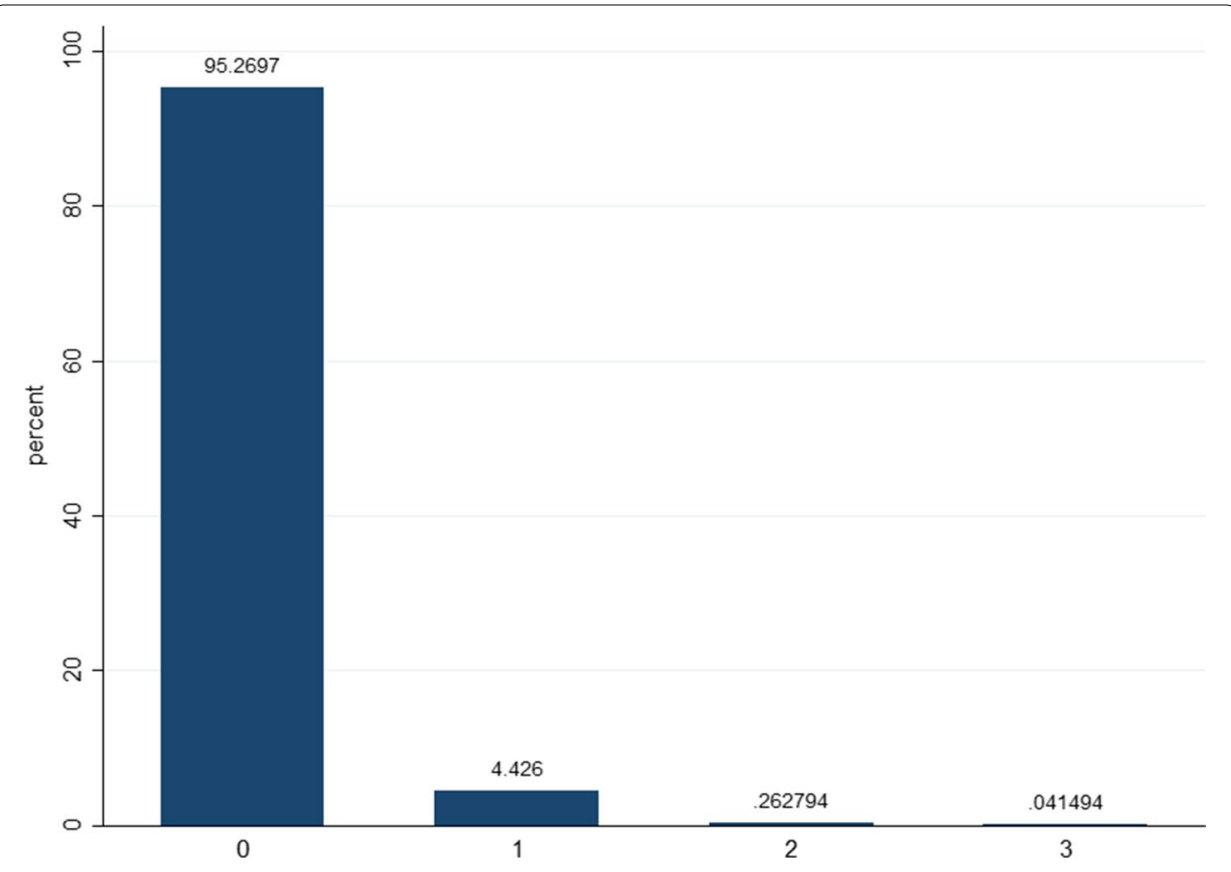

Fig. 1 Distributions (number) of perinatal mortality per mother

expected an over-dispersion of the response variable and this overdispersion coming up due to an excess zero. Because of a large number of zero outcomes, the histogram is highly picked at the very beginning.

\section{Test of over-dispersion}

The over-dispersion parameter test $\mathrm{H} 0: \varnothing=0$, is not statistically significant, which provides evidence for preferring the PLH over the $\mathrm{P}(\mathrm{p}$-value $=0.044)$. Due to this over-dispersion, we applied the Poisson logit hurdle model.

\section{Result of Poisson logit hurdle model}

\section{Count model coefficients}

The expected number of perinatal mortality of a mother whose age 30-39 years were $64 \%$ less likely to experience as compared to whose age less than 30 years keeping the other variable constant. Mothers whose age $40-49$ were $81 \%$ less likely to experience a number of perinatal mortality as compared to their counterparts. The expected number of perinatal mortality of mothers having more than 4 parity were more likely to experience a count of perinatal mortality as compared to less than equal to 4 . The expected number of perinatal mortality of multiple pregnant mothers was more likely risky to a number of perinatal mortality relative to single pregnant.

\section{Zero hurdle model coefficients}

This finding is the estimated Zero hurdle Regression coefficient's odds ratio compared to the group, considering other variables are held constant in the model. The odds of the non-zero perinatal mortality for mothers coming from rural were 1.52 times that 
as compared to urban. The Zero hurdle model indicated that the estimated odds of the number of perinatal mortality for a mother who delivered in the cesarean section was 2.29 times as likely those who give birth normal delivery. As the same wise, the expected non-zero perinatal mortality was significantly affected by preceding birth interval, multiple pregnancies, secondary + husband education, delivered at an institution, and history of abortion (Table 1).

\section{Discussion}

Perinatal mortality is several early neonatal death and stillbirth, which indicates the quality of delivery care and the overall health status of the country [1]. Poisson regression model is used to identify associated factors of count outcomes like perinatal mortality per mother. In the case of zero excessed and over-dispersed data, the Poisson logistics hurdle model is better for count data than the normal Poisson model.

This study aimed to apply the Poisson logistics hurdle model to identify associated factors of perinatal mortality in Ethiopia using 2016 EDHS data. Consequently, the expected counts of perinatal mortality for mothers whose ages 30-39 were less likely risky as compared with those whose ages were less than 30 years. Similarly, mothers whose ages 40-49 were less risky to experience perinatal mortality compared with counterparts. This study is inconsistent with a study conducted in Jima zone, which reported that maternal age has no association with perinatal mortality [20] and a study conducted in Uganda reported that mothers whose age greater than 30 years were more risky to perinatal mortality than their counterparts [21]. The possible reason for this difference may be related to methodological difference, that study used binary logistics regression analysis method, it might lose information when it categorizes binary categories.

Moreover; mothers who have four and above parity were more likely risky to experience perinatal mortality as compared to counterparts. This finding is in line with a study conducted in Sudan, and southwest Ethiopia [22, 23]. Moreover, this finding is inconsistent with a study conducted in Uganda [21]. The possible reason for this variation might be the participant variation from this study is a rural residence and methodological difference. The expected counts of perinatal mortality of mothers who had multiple pregnancies were 6.29 times more likely risky than single pregnant mothers. This result is agreed with a study conducted in southwest Ethiopia, which stated that twin births are increased perinatal mortality [20]. This finding is also consistent with a study conducted in Iran, which revealed that twin pregnancy has no association with perinatal mortality [24]. The reason might be the study design which was retrospective case-control. In addition to the study design, there was methodological statistical analysis, sociodemographic and educational variation.

This study revealed that mothers who lived in rural were 1.52 times as likely to experience none zero perinatal mortality as compared to urban ones. This finding disagreed with a study conducted in Sudan [22]. The main reason for this variation might be the method of data analysis and sample size variation, which is binary logistics.

In this study, we assessed the mode of delivery with a count of perinatal mortality per mother. The odd of a mother who delivered with cesarean section was 2.26 times as likely to experience non-zero perinatal mortality as compared to vaginal delivery. This finding is in line with a study conducted in Iran, Sudan, and Gamo zone Ethiopia 
Table 1 Results of Poisson logistics hurdle model estimation

\begin{tabular}{|c|c|c|c|c|}
\hline Variables & Estimate & ARR $(95 \% \mathrm{Cl})$ & Std. error & $\operatorname{Pr}(>|z|)$ \\
\hline \multicolumn{5}{|c|}{ Count model coefficients (truncated Poisson with log link) } \\
\hline Intercept & -2.61349 & $0.07(0.00,7.45)$ & 2.35790 & 0.267 \\
\hline \multicolumn{5}{|l|}{ Mother age } \\
\hline \multicolumn{5}{|l|}{$<30$} \\
\hline $30-39$ & -1.02022 & $0.36(0.13,1.01)$ & 0.52320 & 0.051 \\
\hline $40-49$ & -1.64377 & $0.19(0.04,0.94)$ & 0.80824 & $0.041^{*}$ \\
\hline \multicolumn{5}{|l|}{ Residence } \\
\hline \multicolumn{5}{|l|}{ Urban } \\
\hline Rural & 0.65787 & $1.93(0.21,17.76)$ & 1.13213 & 0.561 \\
\hline \multicolumn{5}{|l|}{ Education maternal } \\
\hline \multicolumn{5}{|l|}{ Not edu } \\
\hline Primary & -0.41685 & $0.66(0.17,2.57)$ & 0.69445 & 0.548 \\
\hline Secondary+ & 1.37389 & $3.95(0.68,23.05)$ & 0.89985 & 0.126 \\
\hline \multicolumn{5}{|l|}{ Parity } \\
\hline \multicolumn{5}{|l|}{$\geq 4$} \\
\hline$>4$ & 1.08141 & $2.95(1.04,8.33)$ & 0.52993 & $0.041^{*}$ \\
\hline \multicolumn{5}{|l|}{ ANC visit } \\
\hline \multicolumn{5}{|l|}{$<4$} \\
\hline$\geq 4$ & 0.25703 & $1.29(0.43,3.89)$ & 0.56239 & 0.647 \\
\hline \multicolumn{5}{|l|}{ Mode of delivery } \\
\hline \multicolumn{5}{|l|}{ Vaginal } \\
\hline Caesarean section & -12.40982 & $0.00(0.00)$ & 330.5691 & 0.970 \\
\hline Preceding birth interval (month) & -0.02380 & $0.98(0.95,1.00)$ & $\begin{array}{l}8 \\
0.01393\end{array}$ & 0.087 \\
\hline \multicolumn{5}{|l|}{ Number of pregnancy } \\
\hline \multicolumn{5}{|l|}{ Single } \\
\hline Multiple & 1.83840 & $6.29(2.12,18.64)$ & 0.55465 & $0.001^{*}$ \\
\hline \multicolumn{5}{|l|}{ Husband education } \\
\hline \multicolumn{5}{|l|}{ Not } \\
\hline Primary & -0.93219 & $0.39(0.12,1.25)$ & 0.58847 & 0.113 \\
\hline Second + & -0.74307 & $0.48(0.09,2.42)$ & 0.83047 & 0.370 \\
\hline \multicolumn{5}{|l|}{ Place of delivery } \\
\hline \multicolumn{5}{|l|}{ Home } \\
\hline Institution & -0.17346 & $0.84(0.31,2.29)$ & 0.51168 & 0.734 \\
\hline \multicolumn{5}{|l|}{ History abortion } \\
\hline \multicolumn{5}{|l|}{ No } \\
\hline Yes & 0.41699 & $1.52(0.68,3.39)$ & 0.40991 & 0.309 \\
\hline \multicolumn{5}{|c|}{ Zero hurdle model coefficients (binomial with logit link) } \\
\hline Intercept & -3.547676 & $0.03(0.01,0.07)$ & 0.437117 & $<0.001^{*}$ \\
\hline Mother age & & & & \\
\hline$(\operatorname{Ref}<+39)$ & & & & \\
\hline $30-39$ & -0.020371 & $0.98(0.73,1.32)$ & 0.152323 & 0.893 \\
\hline 40-49 & 0.241630 & $1.27(0.83,1.96)$ & 0.220879 & 0.2738 \\
\hline Residence & & & & \\
\hline Urban & & 1 & & \\
\hline Rural & 0.419478 & $1.52(1.03,2.25)$ & 0.200311 & $0.036^{*}$ \\
\hline Maternal education & & & & \\
\hline Not education & & & & \\
\hline Primary & -0.047021 & $0.95(0.71,1.29)$ & 0.153974 & 0.760 \\
\hline Secondary+ & 0.094187 & $1.10(0.65,1.87)$ & 0.270744 & 0.727 \\
\hline
\end{tabular}


Table 1 (continued)

\begin{tabular}{|c|c|c|c|c|}
\hline Variables & Estimate & ARR $(95 \% \mathrm{Cl})$ & Std. error & $\operatorname{Pr}(>|z|)$ \\
\hline \multicolumn{5}{|l|}{ Parity } \\
\hline \multicolumn{5}{|l|}{$<4$} \\
\hline$>4$ & -0.176629 & $0.84(0.62,1.14)$ & 0.156863 & 0.260 \\
\hline \multicolumn{5}{|l|}{ ANC visit } \\
\hline \multicolumn{5}{|l|}{$<4$} \\
\hline$\geq 4$ & -0.113745 & $0.89(0.68,1.17)$ & 0.138690 & 0.4124 \\
\hline \multicolumn{5}{|l|}{ Mode of delivery } \\
\hline \multicolumn{5}{|l|}{ Vaginal } \\
\hline Caesarean section & 0.829849 & $2.29(1.31,4.00)$ & 0.283963 & $0.003^{*}$ \\
\hline Preceding birth interval (month) & -0.015882 & $0.98(0.98,0.99)$ & 0.003059 & $<0.001^{*}$ \\
\hline \multicolumn{5}{|l|}{ Number of pregnancy } \\
\hline \multicolumn{5}{|l|}{ Single } \\
\hline Multiple & 1.827527 & $6.22(3.69,10.4)$ & 0.266511 & $<0.001^{*}$ \\
\hline \multicolumn{5}{|l|}{ Husband education } \\
\hline \multicolumn{5}{|l|}{ Not educated } \\
\hline Primary & -0.179535 & $0.84(0.64,1.09)$ & 0.136903 & 0.189 \\
\hline Second+ & -0.672439 & $0.51(0.33,0.80)$ & 0.228902 & $0.003^{*}$ \\
\hline \multicolumn{5}{|l|}{ Place of delivery } \\
\hline \multicolumn{5}{|l|}{ Home } \\
\hline Institution & 0.338296 & $1.40(1.06,1.86)$ & 0.144161 & $0.018^{*}$ \\
\hline \multicolumn{5}{|l|}{ Abortion } \\
\hline \multicolumn{5}{|l|}{ No } \\
\hline Yes & 2.103379 & $8.19(6.42,10.4)$ & 0.124540 & $<0.001^{*}$ \\
\hline
\end{tabular}

* significance at 0.05

$[22,24,25]$. The odds of experiencing perinatal mortality were decreased by $2 \%$ for a 1 -month increase of the preceding birth interval of the mother. This finding is consistent with a study conducted at Ilu Ababura, Oromia zone, and Addis Ababa in Ethiopia $[26,27]$. The possible reason could be related to the preparedness of a mother in psychology and nutrition aspect variation.

The odds of experiencing none zero perinatal mortality were 6.22 times as likely to occur in multiple pregnancies as compared to single. This finding was supported by [20]. A mother having a secondary and above-educated husband was less likely to perinatal mortality compared with not educated. The reason might be on caring of mothers during deliveries and lactate in terms of nutrition, educated husbands have nutritional knowledge and giving care for mothers and the fetus.

Surprisingly, in this study, the odds of non-zero perinatal mortality were more likely those mothers who delivered an institution as compared to home-delivered. It might be related to the mode of delivery at the institution. This finding disagrees with a study conducted in Sudan. The reason might be a method of analysis and sample size, which used binary logistics regression and the sample size was 808 participants. Mothers who had a history of abortion were 8.19 times as likely to have perinatal mortality compared with no history of abortion. This finding is supported by a study conducted in Iran [24]. The possible reason might be the effect of abortion on the organs of the mother and fetus. 
This study has a contribution to literature by introducing advanced statistical model, which is the Poisson logit hurdle model on count data to investigate the associated factors of perinatal mortality. Several studies were used binary logistic regression model, which lost some important information due to categorization like (yes/ no) and identification of zero type (true zero/false zero) [20, 23, 25].

The strengths of this study were using a recent nationally representative data set. And also, this study used the Poisson logit hurdle model to identify the associated factors of perinatal mortality in Ethiopia. One of the limitations of this study was important clinical factors were not assessed and the design of this study was crosssectional that cannot estimate any causal relationship.

\section{Conclusion}

This study has a contribution to literature by applying advanced Poisson logit hurdle model. The Poisson logit hurdle model is better for the perinatal mortality count data set. Based on the findings of this study, the main protective associated factors were 40-49 years age of mother, having long preceding birth interval, and secondary + husband education. Parity four and above, rural residence, Caesarean section, multiple pregnancies, institutional delivery, having a history of abortion were increased perinatal mortality. The implication of this study that targeted intervention can be designed focusing on family planning and mode of delivery to minimize perinatal mortality in the country.

\section{Abbreviations}

Cl: Confidence interval; EDHS: Ethiopia Demography and Health Survey; MLE: Maximum Likelihood Estimation; OR: Odds ratio; ZIP: Zero Inflated Poisson; PLH: Poisson logit Hurdle; PM: Perinatal mortality; PMR: Perinatal mortality rate.

\section{Acknowledgements}

The authors would like to acknowledge the Measure Demographic and Health Survey for providing online permission to use the Ethiopia Demographic and Health Survey 2016 data set.

\section{Authors' contributions}

All authors (AGA, and AMA) were responsible for the formulation of the methodology. All authors (AGA, MSW, and AMA) made substantial contributions to the conception and design of the study, analyzed and interpreted the data, and was major contributor in writing the manuscript. MSW and AGA drafted the manuscript. All authors read and approved the final manuscript.

Funding

No funding was received for this study.

\section{Availability of data and materials}

The data that support the findings of this study are available from the Measure DHS website (www.measuredhs.com but restrictions apply to the availability of these data, which were used under license for the current study, and so are not publicly available. Data are however available from the authors upon reasonable request and with permission of Measure DHS.

\section{Declarations}

Ethics approval and consent to participate Not applicable.

Consent for publication

Not applicable.

Competing interests

The authors declare that they have no competing interests. 


\begin{abstract}
Author details
${ }^{1}$ Department of Statistics, College of Natural and Computational Science, Debre Markos University, Debre Markos, Ethiopia. ${ }^{2}$ Department of Epidemiology and Biostatistics, School of Public Health, College of Medicine and Health Science, Bahir Dar University, Bahir Dar, Ethiopia. ${ }^{3}$ Department of Mathematical and Statistical Modeling (Statistics), Bahir Dar Institute of Technology-Bahir Dar University, Bahir Dar, Ethiopia.
\end{abstract}

Received: 30 August 2021 Accepted: 26 January 2022

Published online: 10 February 2022

\title{
References
}

1. Killewo J, Heggenhougen K, Quah SR. Epidemiology and demography in public health. Cambridge: Academic Press; 2010.

2. Organization WH. The WHO application of ICD-10 to deaths during the perinatal period: ICD-PM. Geneva: Organization $\mathrm{WH} ; 2016$.

3. Quah SR. International encyclopedia of public health. Cambridge: Academic Press; 2016.

4. Allanson ER, Tunçalp Ö, Gardosi J, Pattinson RC, Francis A, Vogel JP, et al. The WHO application of ICD-10 to deaths during the perinatal period (ICD-PM): results from pilot database testing in South Africa and United Kingdom. BJOG Int J Obstet Gynaecol. 2016;123(12):2019-28.

5. Akombi BJ, Renzaho AM. Perinatal mortality in sub-Saharan Africa: a meta-analysis of demographic and health surveys. Ann Glob Health. 2019;85(1):106.

6. Organization WH. Reaching the every newborn national 2020 milestones: country progress, plans and moving forward. Geneva: Organization WH; 2017.

7. Organization WH. Neonatal and perinatal mortality: country, regional and global estimates. Geneva: World Health Organization; 2006.

8. Jang H, Lee S, Kim SW. Bayesian analysis for zero-inflated regression models with the power prior: Applications to road safety countermeasures. Accid Anal Prev. 2010;42(2):540-7.

9. Prasetijo J, Musa WZ, editors. Modeling zero-inflated regression of road accidents at Johor Federal Road F001. In: MATEC web of conferences; 2016: EDP Sciences.

10. Hilbe JM. Negative binomial regression. Cambridge: Cambridge University Press; 2011.

11. Hofstetter H, Dusseldorp E, Zeileis A, Schuller AA. Modeling caries experience: advantages of the use of the hurdle model. Caries Res. 2016;50(6):517-26.

12. Sarul LS, Sahin S. An application of claim frequency data using zero inflated and hurdle models in general insurance. J Bus Econ Finan. 2015:4(4):732-43.

13. Lambert D. Zero-inflated Poisson regression, with an application to defects in manufacturing. Technometrics. 1992;34(1):1-14.

14. Min Y, Agresti A. Random effect models for repeated measures of zero-inflated count data. Stat Model. 2005:5(1):1-19.

15. Feng CX. A comparison of zero-inflated and hurdle models for modeling zero-inflated count data. J Stat Distrib Appl. 2021;8(1):1-19.

16. Hilbe JM. Modeling count data. Cambridge: Cambridge University Press; 2014.

17. Sharker S, Balbuena L, Marcoux G, Feng CX. Modeling socio-demographic and clinical factors influencing psychiatric inpatient service use: a comparison of models for zero-Inflated and overdispersed count data. BMC Med Res Methodol. 2020:20(1):1-10.

18. Lam K, Xue H, Bun CY. Semiparametric analysis of zero-inflated count data. Biometrics. 2006;62(4):996-1003.

19. Hilbe JM, De Souza RS, Ishida EE. Bayesian models for astrophysical data: using R, JAGS, Python, and Stan. Cambridge: Cambridge University Press; 2017.

20. Debelew GT. Magnitude and determinants of perinatal mortality in Southwest Ethiopia. J Pregnancy. 2020. https:// doi.org/10.1155/2020/6859157.

21. Arach AAO, Tumwine JK, Nakasujja N, Ndeezi G, Kiguli J, Mukunya D, et al. Perinatal death in Northern Uganda: incidence and risk factors in a community-based prospective cohort study. Glob Health Action. 2021;14(1):1859823.

22. Ali AAA, Elgessim ME, Taha E, Adam GK. Factors associated with perinatal mortality in Kassala, Eastern Sudan: a community-based study 2010-2011. J Trop Pediatr. 2014;60(1):79-82.

23. Aragaw Y. Perinatal mortality and associated factor in Jimma university specialized hospital South West Ethiopia. Gynecol Obstet (Sunnyvale). 2016;6(409):2161-932.

24. Hasheminasab L, Ranaei F, Majidi J, Zaheri F, Karime R. Risk factors associated with perinatal mortality at Besat Hospital in Sanandaj in 2013-2015: a case-control study. J Midwifery Reprod Health. 2021;9(2):2690-7.

25. Dessu S, Dawit Z. Perinatal mortality and associated factors among antenatal care attended pregnant mothers at public hospitals in Gamo Zone, Southern Ethiopia. Front Pediatr. 2020;8:869.

26. Geda A, Shemsu S, Debalke R. Determinants of perinatal mortality in public hospitals of Iluu Abbaa Boor Oromia Region, South West Ethiopia, 2019: unmatched case-control study. Res Rep Neonatol. 2021;11:57-66.

27. Getiye Y, Fantahun M. Factors associated with perinatal mortality among public health deliveries in Addis Ababa, Ethiopia, an unmatched case control study. BMC Pregnancy Childbirth. 2017;17(1):1-7.

\section{Publisher's Note}

Springer Nature remains neutral with regard to jurisdictional claims in published maps and institutional affiliations. 